\title{
Women in Advanced Reproductive Age: Are the Follicular Output Rate, the Follicle-oocyte Index and the Ovarian Sensitivity Index Predictors of Live Birth in an IVF Cycle?
}

\section{Andrea Roberto Carosso ( $\square$ andrea88.carosso@gmail.com )}

Università degli Studi di Torino https://orcid.org/0000-0002-7734-4499

\section{Rik van EEKELEN}

Amsterdam UMC - Locatie AMC: Amsterdam UMC Locatie AMC

Alberto Revelli

Università degli Studi di Torino: Universita degli Studi di Torino

\section{Stefano Canosa}

Università degli Studi di Torino: Universita degli Studi di Torino

Noemi Mercaldo

Università degli Studi di Torino: Universita degli Studi di Torino

\section{Chiara Benedetto}

Università degli Studi di Torino: Universita degli Studi di Torino

\section{Gianluca Gennarelli}

Università degli Studi di Torino: Universita degli Studi di Torino

\section{Research}

Keywords: IVF, unexplained infertility, follicular output rate, follicle-oocyte index, ovarian sensitivity index, ovarian responsiveness, live birth

Posted Date: September 7th, 2021

DOl: https://doi.org/10.21203/rs.3.rs-868149/v1

License: (c) (i) This work is licensed under a Creative Commons Attribution 4.0 International License. Read Full License

Version of Record: A version of this preprint was published at Journal of Clinical Medicine on February 6th, 2022. See the published version at https://doi.org/10.3390/jcm11030859. 


\section{Abstract}

\section{Background}

Several researches have investigated alternative markers related to ovarian responsiveness in order to better predict IVF outcomes, particularly in advanced reproductive aged women. The follicular output rate (FORT), the follicle-oocyte index (FOI) and the ovarian sensitivity index (OSI) are among the most promising. However, these three metrics have not been investigated as independent predictors of live birth in women of advanced reproductive age, neither have been compared to the two 'component' characteristics that were used to calculate them.

\section{Methods}

A logistic regression model containing all relevant predictors of ovarian reserve or response was used to evaluate the potential of FORT, FOI and OSI as predictors of live birth. After, the non-linear associations between FORT, FOI and OSI and the probability of live birth was evaluated. Finally, we fitted multiple logistic regression models to compare whether FORT, FOI and OSI were more informative predictors than the two 'components' used to calculate them.

\section{Results}

590 couples received a total of 740 IVF cycles, after which $127(17.5 \%)$ obtained a live birth. None of FORT, FOI and OSI showed a strength of association or a p-value even close to female age (Odds ratio for live birth (95\% Confidence Interval) 1.00 (0.99-1.01), 1.00 (0.99-1.01), 0.98 (0.88-1.11) and 0.58 (0.480.72 ), respectively). The three models comparing FORT, FOI and OSI with the two 'components' used to calculate them were not more informative.

\section{Conclusion(s)}

In a population of women of advanced age with unexplained infertility, none of FORT, FOI and OSI were predictive of live birth and more predictive than the two 'components' characteristics that were used to calculate them. We suggest clinicians and researchers still to use female age as the most reliable predictor of an IVF treatment.

\section{Trial registration}

retrospectively registered

\section{Background}

The number of retrieved oocytes is considered one of the most important predictors of live birth in an in vitro fertilization (IVF) cycle [1-3]. Oocytes yield depends on several factors that modulate the ovarian responsiveness to controlled ovarian stimulation (COS). These include the type and dose of exogenous 
gonadotropins, the intrinsic sensitivity of the ovary to hormonal stimulation, partially correlated to the polymorphic variants of the FSH-receptor [4], and the rhythm of follicular maturation waves [5].

The individual variability of these intrinsic factors may be associated with an unexpectedly low ovarian response during COS. Thus, the total number of retrieved oocytes does not always accurately reflect the ovarian potential. The wish to find the true ovarian potential in terms of the maximum number of retrieved oocytes and to link this to aforementioned factors has stimulated the research for more sophisticated markers of ovarian function and response, such as the ovarian sensitivity index (OSI) [6, 7], the follicular output rate (FORT) [8, 9] and the follicle-oocyte index (FOI) [10]. The OSI is defined as the ratio between the number of retrieved oocytes and the total dose of FSH administered; the FORT represents the ratio between the number of pre-ovulatory follicles obtained after COS and the prestimulation pool of small antral follicles; the FOI is the ratio between the number of retrieved oocytes and the number of antral follicles at the beginning of COS.

The OSI represents the ovarian sensitivity to exogenous gonadotropins and since its introduction it has been used to adjust the COS regimen in subsequent IVF cycles, whereas FORT and FOI have been claimed to be a good representation of the dynamic nature of follicular growth and the follicular competence. As a matter of fact, several studies have shown that these markers correlate positively to IVF outcome $[8,11$, 12].

However, the aforementioned markers are also affected by some limitations: it is unlikely that all three metrics have simple linear associations with the probability of live birth. The OSI does not take into account the gonadotropin regimen nor it consider the type of gonadotropin (recombinant, urinary, with LH-like activity or not). Furthermore, it might be misleading if inappropriate low starting doses of exogenous gonadotrophins are given. Neither FORT nor FOI assess the total number of mature oocytes retrieved, whereas both indexes depend on the baseline antral follicle count (AFC). AFC on itself shows limited predictive value in older women, whose follicles are affected by increased granulosa cell apoptosis, impaired mitochondrial function and increased oxidative stress [13, 14]. Indeed, older women represent the class of patients in whom it is more difficult to predict the chances of IVF success. In this context, the use of multiple surrogate markers that indicate ovarian reserve from different perspectives appears promising.

However, no studies have so far considered these three metrics (FORT, FOI and OSI) as independent predictors and compared metrics to the two 'component' characteristics that were used to calculate them. In this retrospective study among women aged 38 or above, we first evaluated non-linear associations between metrics and live birth in women of advanced reproductive age, then compared all three metrics to one another and finally, compared these three metrics to the two variables that were used to calculate them.

\section{Methods}




\section{Patient Population}

The study was performed in accordance with the Helsinki Declaration and with approval of the Institutional Review Board. In this retrospective analysis we included a cohort of women aged 39 years or above, affected by unexplained infertility, who were admitted to the IVF unit of the S. Anna academic hospital between 2010-2019. Only autologous cycles were considered. Exclusion criteria were: female body mass index (BMI: $\mathrm{Kg} / \mathrm{m} 2)>32 \mathrm{~kg} / \mathrm{m} 2$, anti-Mullerian hormone $(\mathrm{AMH})<0.1$ and/or early follicular phase follicle stimulating hormone $(\mathrm{FSH})>20 \mathrm{UI} / \mathrm{l}$, any known cause of female infertility (i.e. previous history of pelvic inflammatory disease, positive anti-Chlamydia IgG, endometriosis, anovulation, etc), cycles with pre-implantation genetic diagnosis. The BMI criterion is imposed by regional legislation in order to reduce the risk of comorbidities related to obesity in pregnancy. All women included in the study had ovulatory cycles and patency of at least one fallopian tube at sonosalpingography (SSG), whereas all male partners had normal basic semen parameters according to the indications of the World Health Organization (WHO), 2010.

\section{ART Procedure}

Controlled ovarian stimulation (COS) was performed either with recombinant FSH (rFSH), human Menopausal Gonadotropin (HP-hMG) or rFSH plus recombinant luteinizing hormone (rLH), under pituitary suppression applying both long and short protocols. The choice of the starting gonadotropin dose was based on age, AMH concentrations, AFC as well as on the response to previous $\mathrm{COS}$. The long protocol was performed administering buserelin (Suprefact, Hoechst, Frankfurt, Germany; 900 mcg/d intranasally) starting from the late luteal phase of the previous cycle. Pituitary suppression was verified after approximately two weeks (appearance of a menstrual bleeding, serum estradiol $<50 \mathrm{pg} / \mathrm{mL}$, endometrial thickness $<3 \mathrm{~mm}$ ) before starting COS. In the short protocol, either the $\mathrm{GnRH}$-antagonist cetrorelix (Cetrotide, Merck, Darmstadt, Germany) or ganirelix (Orgalutran fi, Merck Sharp \& Dome, Kenilworth, NJ, USA) were started at a subcutaneous dose of $0.25 \mathrm{mg} / \mathrm{d}$ according to a flexible schedule, when at least one follicle $\geq 12 \mathrm{~mm}$ in mean diameter was observed at ultrasound (US).

COS was monitored by serial transvaginal US and serum estradiol (E2) measurements performed every second day from stimulation day 6-7. COS continued until at least two follicles reached $18 \mathrm{~mm}$ in mean diameter, when ovulation was triggered by injecting either 10,000 international units (IU) of hCG (Gonasi HPfi, IBSA, Lugano, Switzerland) or 250 mcg of rHCG (Ovitrellefi, Merck, Darmstadt, Germany) subcutaneously. US-guided transvaginal oocyte aspiration (OPU) was performed approximately $36-37 \mathrm{~h}$ after hCG administration, under local anesthesia (paracervical block). Oocytes were immediately recovered from the follicular fluid, then washed in buffered medium and stored until fertilization procedure.

Semen samples were examined to assess sperm concentration, motility, and morphology according to the WHO guidelines [15]. Samples were then prepared by density gradient centrifugation in order to select motile, morphologically normal spermatozoa. Conventional IVF or ICSI were performed on all available 
oocytes within 4 hours after oocytes collection or after $2 \mathrm{~h}$ from cumulus cells removal, respectively. After 16-18 hours of incubation in controlled atmosphere, the occurrence of normal fertilization was assessed.

\section{Embryo Selection and Transfer}

Zygotes were placed in pools in 4-wells dish (Thermo Scientific, Roskilde, Denmark) and embryos were cultured in pre-equilibrated Cleavage medium (Cook) overlain with mineral oil using incubators (Minc, COOK, Bloomington, IN, USA) in a gas phase consisting of $5 \% 02,6-7 \% \mathrm{CO} 2$, balanced with $\mathrm{N} 2$.

All cleaved embryos were morphologically evaluated under a conventional stereomicroscope, using the IMCS score by Holte [16].

Embryos(s) transfer was performed using a soft catheter, under ultrasound guidance. According to the policy of our IVF unit during the time period under study, one or two embryos were transferred on day 2/3 post-fertilization. Only embryos reaching the blastocyst stage were vitrified and thawed in subsequent cycles. The luteal phase was supported by administering $180 \mathrm{mg}$ /day natural progesterone (Crinone $8 \mathrm{fi}$, Merck,Darmstadt, Germany) for 15 days.

Pregnancy was assessed by serum hCG assay after 15 days from embryo transfer (ET) and then confirmed if at least one gestational sac was visualized on transvaginal US after two further weeks.

Live birth was defined as the delivery of a live-born infant ( $>24$ weeks of gestation).The cLBR was defined as live deliveries (at least one live birth) per women including both fresh and frozen/thawed embryo transfers obtained from the same cycle.

\section{Metrics}

FORT was defined as the number of pre-ovulatory follicles of at least $15 \mathrm{~mm}$ in response to ovarian stimulation divided by the pre-treatment AFC (which included all follicles at least $11 \mathrm{~mm}$ in mean diameter) multiplied by 100 .

FOI was defined as the number of oocytes collected after ovarian stimulation divided by the pre-treatment AFC multiplied by 100 . For agonist cycles, the AFC was evaluated as previously described [8].

OSI was defined as the number of oocytes collected after ovarian stimulation divided by the total dose of FSH administered, per 1000.

\section{Statistical analyses}

First, we fitted a logistic regression model containing all relevant predictors of ovarian reserve or response to evaluate whether these common indicators were 'independent' predictors of live birth: female age, duration of infertility, previous ART, primary or secondary subfertility, preovulatory follicles, oocytes collected, AFC and total FSH dose. Since only age was found to be a strong predictor of live birth, we evaluated the non-linear associations between the three metrics separately (FORT, FOI and OSI) and the estimated probability of live birth after IVF to get an indication of the strength and functional form of the 
associations. To this end, we fitted logistic regression models on live birth after IVF using restricted cubic splines with 4 knots for the three mentioned metrics, and then used these models to predict the probability of a live birth after IVF for a range of values for each metric. We plotted the results using simultaneous $95 \%$ Cls that take into account the many comparisons drawn in these plots.

Next, we fitted multiple logistic regression models to compare whether FORT, FOI and OSI were more informative predictors than the two 'components' used to calculate them. For FORT, we compared a model containing FORT to a model containing preovulatory follicles and AFC. For FOI, we compared a model containing FOI to a model containing oocytes collected and AFC. For OSI, we compared a model containing OSI to a model containing oocytes collected and total FSH dose. In addition, the following four factors were added to all models as they were considered the most important and could explain part of the associations of the three metrics and/or their components: female age, duration of infertility, previous ART and primary or secondary subfertility.

We also assessed whether the associations between FORT, FOI and OSI were different for poor responders (according to Bologna criteria [17]) to stimulation (defined as $\mathrm{AMH}<1.1$ or AFC $<7$ ) by including an interaction between the predictor and a factor denoting whether a woman was a poor responder.

We determined the best fitting and most informative model in terms of the lowest Akaike Information Criterion (AIC) [18]. For all models, we used a robust standard error that allows for clustering on patient ID to adjust precision for the fact that couples can receive multiple cycles [19].

\section{Sample size calculation and software}

Using the '10 events per variable' rule of thumb, we would be able to include approximately 11-13 predictors in our model(s) [20]. Using a more elaborate, contemporary method, we calculated that with 10 candidate predictors and approximately $18 \%$ who had the event, 700 participants are required to get an accuracy of 0.05 in the mean average percentage error [21].

Data was compiled in Excel and analysed in R 3.6.0 using the $r m s$, mice, xtable and dplyr packages (R Core Team, 2017).

\section{Results}

Data on 590 couples were available. These 590 couples received a total of 740 IVF cycles, after which 127 (17.5\%) obtained a live birth. The baseline characteristics are shown in Table 1. 
Table 1

Baseline characteristics for all 590 couples.

\begin{tabular}{|ll|}
\hline Baseline characteristics & Mean or median and 2.5th-97.5th percentile \\
\hline Female age at treatment (years) & $40.8(39.0-42.0)$ \\
\hline Male age at treatment (years) & $42.0(33.0-53.0)$ \\
\hline Duration of infertility (median, years) & $2.0(1.0-9.3)$ \\
\hline Percentage of progressive motile sperm (median) & $40(32-48)$ \\
\hline AMH (median) & $1.1(0.1-6.9)$ \\
\hline FSH total dose, UI & $3062(1018-5850)$ \\
\hline AFC (median) & $10(2-30)$ \\
\hline OSI (median) & $1.8(0.3-11.1)$ \\
\hline FORT (median) & $50(14-165)$ \\
\hline FOI (median) & $57(13-175)$ \\
\hline Number of preovulatory follicles (median) & $5(1-15)$ \\
\hline Number of oocytes collected (median) & $5(1-18)$ \\
\hline Infertility (primary versus secondary) & $363(62 \%)$ \\
\hline Smoking (yes versus no) & $69(12 \%)$ \\
\hline Poor responders (yes versus no) & $326(55 \%)$ \\
\hline Previous ART & $187(28.3 \%)$ \\
\hline None & $238(40.3 \%)$ \\
\hline Only IUI & $185(31.4 \%)$ \\
\hline IVF & $167)$ \\
\hline
\end{tabular}

In Table 2 we reported the fully adjusted model, showing associations between patient characteristics and the odds of a live birth after IVF. After adding all predictors, only female age $(p<0.001)$ was a significant predictor of live birth after IVF (Table 2). None of the metrics (FORT, FOI and OSI) showed a strength of association or a p-value even close to female age, even considering the different scales they are on. 
Table 2

Associations between patient characteristics and the odds of a live birth after IVF.

\begin{tabular}{|ll|}
\hline Patient characteristics & Odds ratio for live birth (95\% CI) \\
\hline Female age at treatment (years) & $0.58(0.48-0.72)$ \\
\hline Duration of infertility (median, years) & $0.93(0.84-1.03)$ \\
\hline AFC (median) & $0.98(0.94-1.03)$ \\
\hline FSH UI, total dose & $1.00(0.98-1.02)$ \\
\hline Number of preovulatory follicles (median) & $1.06(0.96-1.17)$ \\
\hline Number of oocytes collected (median) & $1.05(0.94-1.17)$ \\
\hline Infertility (primary versus secondary) & $0.89(0.58-1.37)$ \\
\hline Poor responders (yes versus no) & $0.84(0.50-1.41)$ \\
\hline Previous ART & \\
\hline Only IUI versus none & $0.74(0.42-1.33)$ \\
\hline IVF versus none & $1.23(0.79-1.93)$ \\
\hline OSI (median) & $0.98(0.88-1.11)$ \\
\hline FORT (median) & $1.00(0.99-1.01)$ \\
\hline FOI (median) & $1.00(0.99-1.01)$ \\
\hline
\end{tabular}

Non-linear associations between metrics and live birth after IVF

Figures 1 through 3 show the non-linear associations between live birth and FORT, FOI and OSI, respectively. For all metrics, we see that higher values were associated with a higher estimated probability of a live birth after IVF. For FORT and FOI this seems to be somewhat linear over the range of their possible values from 0 to 100 , but for OSI the association seems to be non-linear. We therefore decided to use the non-linear fit for the comparison with its two components in the next analysis.

Comparing metrics to their two components

The model with FORT was not more informative than the model with preovulatory follicles and AFC as shown by a 5-point higher AIC for the former. The model with FOI was not more informative than the model with oocytes collected and preovulatory follicles as shown by a 5-point higher AIC for the former. The model with OSI modelled as non-linear was not more informative than the model with oocytes collected and FSH dose as shown by a 4-point higher AIC for the former. 
The associations between live birth and FORT, FOI and OSI were not significantly different in poor responders to normal responders ( $p$-values for interactions $0.744,0.151$ and 0.995 ).

\section{Discussion}

In this retrospective analysis the predictive value of obtaining a live birth of three IVF indicators has been evaluated in elderly women of couples affected by unexplained infertility: FOI, FORT and OSI do not show a stronger or more informative association with live birth than the components used for their calculation, i.e. the number of oocytes retrieved, the AFC, the number of preovulatory follicles and the FSH total dose. Female age remained the most reliable predictor for live birth in an IVF cycle.

Women of advanced reproductive age remain an open dilemma and a challenge for all clinicians working in the field of assisted reproductive technologies (ART).

Over the years, several attempts have been made, in order to identify surrogate markers of ovarian reserve, which in turn could be markers of IVF outcomes. This would allow to decide for whom treatment is expected to be (cost-)beneficial. However, so far studies on the association between markers such as $\mathrm{AMH} / \mathrm{AFC}$ and implantation, pregnancy, and/or live birth after assisted conception reported conflicting results $[22,23]$.

Therefore, several authors have investigated alternative markers that are discussed in this paper, particularly those related to ovarian responsiveness to COS, in order to better predict IVF outcomes. Efficient markers would be of particular interest in subgroups of low prognosis patients defined both by the Poseidon and the Bologna criteria $[17,24]$.

Alviggi et al. analyzed the predictive role of FOl to assess ovarian sensitivity in hypo-responders patients [10]. The authors concluded that FOI might reflect the dynamics of follicular growth in response to exogenous gonadotropin, better than traditional markers of ovarian reserve. In particular, low FOI values imply that only a fraction of available antral follicles was exploited during COS, suggesting that there might be therapeutic opportunities (increasing the FSH dose and/or adding $\mathrm{LH}$ ) to improve ovarian responsiveness, and therefore the overall prognosis.

Grynberg et al. discussed the potential use of FORT as a quantitative and qualitative marker of ovarian responsiveness to gonadotropins, and the possible implications for the applicability of the Poseidon criteria [25]. They stated that FORT may reveal impaired sensitivity to FSH and should be used to guide the decision of treatment protocol, gonadotropin, and stimulation doses to be used for hypo-responders.

Recently, the amount of hormone medication needed for each oocyte produced (i.e. the OSI) was investigated in a retrospective cohort study that included more than 1200 women undergoing IVF with FSH/hMG stimulation [26]. Consistently with previous results in younger women with excellent pregnancy potential [7], the OSI was found to be predictive of pregnancy and live birth also in older women with a more unfavorable prognosis [26]. The authors concluded that OSI could be employed in counseling 
women of advanced age about their reproductive potential, bridging the gap between the purely quantitative aspect of ovarian reserve and the more qualitative approach of ovarian competence.

So far, no study has been published on whether these surrogates offer significantly higher performance than their constituent parameters, particularly in women of advanced age. To be specific: are FORT and FOI more predictive of baseline AFC, the number of pre-ovulatory follicles and the number of oocytes retrieved? As for the OSI, is it more informative than the number of oocytes and the total dose of FSH used?

Despite the limitations of a retrospective study, our results seem to scale back the capabilities of FORT, $\mathrm{FOI}$ and OSI to answer the above questions. The combination of multiple indicators of ovarian reserve and ovarian response to COS (FOI and FORT) or in term of FSH administered (OSI) does not seem to be more advantageous than the traditional predictors of IVF outcomes. In addition, most of these predictive factors only become available after at least one IVF cycle was conducted, limiting their usage in clinical decision-making on starting IVF. Thus, there is still a knowledge gap regarding the possibility to predict the oocyte quality, especially using only information that is available before starting COS.

In a cohort of couples with unexplained infertility and advanced female age, only age was found to be a clear predictor of live birth after IVF, further confirming the intrinsic awareness of every IVF expert: the age of the egg is what really matters. This conclusion is in agreement with what has already been reported in previous studies $[22,27,28]$.

Indeed, numerous factors are implicated in the final outcome of an IVF treatment: oocyte and embryo quality, endometrial receptivity, woman general health conditions, etc. Probably, only the female age is able to coherently capture all these factors. The impact of age per se seems truly relevant, considering that the correlation with IVF outcome is stronger than that of all markers considered or even combined, in spite of a rather narrow distribution of ages.

Note that FORT, FOI, and OSI can be considered markers that indicate different aspects of the response to COS; we merely showed that their association with live birth after IVF are negligible when considering female age and their constituent parameters.

This result could have more than one explanation. First of all, one should consider that each of the parameters used for the calculation of the three metrics are operator- dependent. The follicle count, which registered a reduction in inter-individual variability with the introduction of 3D technology [29], is still widely evaluated in 2D in most of the centers, as in the present study. Such a variable is inevitably affected by the skill and the accuracy of the operator. Similarly, there is no unanimous agreement on which should be the starting dose of gonadotropins in an IVF treatment [30,31]. Whereas several algorithms have been developed over the years [32, 33], the starting dose is still widely established on the basis of the operator's clinical sensitivity with respect to multiple parameters (AMH, AFC, age, body mass, previous COS etc.). Obviously, the total dose of gonadotropins administered is partly affected by the initial decision. 
Also the number of oocytes retrieved can o be affected by the experience of the operator [34].

In summary, all of these parameters suffer from measurement error, opening up the possibility of adding more noise i.e. (non-)random variability. By adding in a model more variables that are operator-dependent it is not surprising that the accuracy of these surrogates is reduced with respect to the single parameter that underlies it. This could also explain why a simple but perfect measure such as female age seems to be more informative.

The main strength of our retrospective analysis is that it represents the first study to investigate whether a non-linear association exists between the metrics FORT, FOI and OSI and LBR in the same homogeneous population, i.e. women of advanced age with unexplained infertility, thus avoiding all the hypothetical confounding factors deriving from all the other plausible causes of infertility (male infertility, endometriosis, tubal obstruction, etc.). In addition, we carefully assessed the fit of models with the constituent parameters using contemporary statistical methods, adjusting for multiple comparisons and adjusting standard errors for couples receiving multiple cycles.

In conclusion, none of the three predictors for fertility that were proposed in literature (FORT, FOI and OSI) were more predictive than the two 'components' characteristics that were used to calculate them. This was in a population of women of advanced age (39 years or above) with unexplained infertility. Nonetheless, in light of a lack of evidence that backs up using the three metrics, we suggest that clinicians and researchers still to using the components themselves (i.e. the number of oocytes collected, the number of preovulatory follicles, AFC and FSH dose) in counselling and prediction modelling.

\section{Abbreviations}

FORT: follicular output rate

FOl: the follicle-oocyte index

OSI: the ovarian sensitivity index

IVF: in vitro fertilization

ART: Assisted reproductive technology

HSSG: hysterosonosalpingography

WHO: world health organization

IgG: Immunoglobulin G

FSH: follicle stimulating hormone

AMH: anti-Mullerian hormone 
BMI: body mass index

COS: controlled ovarian stimulation

rFSH: recombinant FSH

HP-hMG: human menopausal gonadotropin

rLH: recombinant luteinizing hormone

AFC: antral follicular count

Gn-RH: gonadotropin-releasing hormone

US: ultrasound

E2: estradiol

IU: international units

hCG: human chorionic gonadotropin

ICSI: intracytoplasmic sperm injection

IMCS: integrated morphology cleavage score

ET: embryo transfer

CLBR: cumulative live birth rate

Cl: confidence interval

AIC: akaike's Information Criterion

\section{Declarations}

Ethics approval and consent to participate:

The study was performed in accordance with the Helsinki Declaration and with approval of the City of Health and Science Institutional Review Board Review Board (n. 0040486, 23/04/2020).

Consent for publication:

not applicable

Availability of data and materials: 
The datasets used and/or analysed during the current study are available from the corresponding author on reasonable request.

\section{Competing interests:}

The authors report no conflict of interest

\section{Funding:}

This research did not receive any specific grant from funding agencies in the public, commercial, or notfor-profit sectors.

\section{Authors' contribution:}

Andrea Carosso conceived the study and wrote the manuscript. Rik van Eekelen performed the data analysis and co-wrote the manuscript. Alberto Revelli contributes to interpretation of the data and critically revised the manuscript. Stefano Canosa and Noemi Mercaldo collect the data and contributes to data analysis. Chiara Benedetto and Gianluca Gennarelli contribute to conceive the study, coordinate the study and critically revised the manuscript. All authors reviewed and approved the final version of the manuscript.

\section{Acknowledgements:}

We thank all the staff of our IVF center for the kind collaboration.

\section{References}

1. Fatemi HM, Doody K, Griesinger G, Witjes H, Mannaerts B. High ovarian response does not jeopardize ongoing pregnancy rates and increases cumulative pregnancy rates in a $\mathrm{GnRH}$-antagonist protocol. Hum Reprod. 2013;28:442-52.

2. Li HWR, Lee VCY, Lau EYL, Yeung WSB, Ho PC, Ng EHY. Role of baseline antral follicle count and antiMullerian hormone in prediction of cumulative live birth in the first in vitro fertilisation cycle: a retrospective cohort analysis. PLoS One. 2013;8:e61095.

3. Sunkara SK, Khalaf Y, Maheshwari A, Seed P, Coomarasamy A. Association between response to ovarian stimulation and miscarriage following IVF: an analysis of 124351 IVF pregnancies. Hum Reprod. 2014;29:1218-24.

4. La Marca A, Sighinolfi G, Argento C, Grisendi V, Casarini L, Volpe A, et al. Polymorphisms in gonadotropin and gonadotropin receptor genes as markers of ovarian reserve and response in in vitro fertilization. Fertil Steril. 2013;99:970-978.e1.

5. Baerwald AR, Adams GP, Pierson RA. Characterization of ovarian follicular wave dynamics in women. Biol Reprod. 2003;69:1023-31. 
6. Biasoni V, Patriarca A, Dalmasso P, Bertagna A, Manieri C, Benedetto C, et al. Ovarian sensitivity index is strongly related to circulating $\mathrm{AMH}$ and may be used to predict ovarian response to exogenous gonadotropins in IVF. Reprod Biol Endocrinol. 2011;9:112.

7. Huber M, Hadziosmanovic N, Berglund L, Holte J. Using the ovarian sensitivity index to define poor, normal, and high response after controlled ovarian hyperstimulation in the long gonadotropinreleasing hormone-agonist protocol: suggestions for a new principle to solve an old problem. Fertil Steril. 2013;100:1270-6.

8. Gallot V, Berwanger da Silva AL, Genro V, Grynberg M, Frydman N, Fanchin R. Antral follicle responsiveness to follicle-stimulating hormone administration assessed by the Follicular Output RaTe (FORT) may predict in vitro fertilization-embryo transfer outcome. Hum Reprod. 2012;27:106672.

9. Genro VK, Grynberg M, Scheffer JB, Roux I, Frydman R, Fanchin R. Serum anti-Müllerian hormone levels are negatively related to Follicular Output RaTe (FORT) in normo-cycling women undergoing controlled ovarian hyperstimulation. Hum Reprod. 2011;26:671-7.

10. Alviggi C, Conforti A, Esteves SC, Vallone R, Venturella R, Staiano S, et al. Understanding Ovarian Hypo-Response to Exogenous Gonadotropin in Ovarian Stimulation and Its New Proposed MarkerThe Follicle-To-Oocyte (FOI) Index. Front Endocrinol [Internet]. Frontiers; 2018 [cited 2020 May 16];9. Available from: https://www.frontiersin.org/articles/10.3389/fendo.2018.00589/full\#B13

11. Chen L, Wang H, Zhou H, Bai H, Wang T, Shi W, et al. Follicular Output Rate and Follicle-to-Oocyte Index of Low Prognosis Patients According to POSEIDON Criteria: A Retrospective Cohort Study of 32,128 Treatment Cycles. Front Endocrinol (Lausanne). 2020;11:181.

12. Revelli A, Gennarelli G, Biasoni V, Chiadò A, Carosso A, Evangelista F, et al. The Ovarian Sensitivity Index (OSI) Significantly Correlates with Ovarian Reserve Biomarkers, Is More Predictive of Clinical Pregnancy than the Total Number of Oocytes, and Is Consistent in Consecutive IVF Cycles. J Clin Med. 2020;9.

13. Bessow C, Donato R, de Souza T, Chapon R, Genro V, Cunha-Filho JS. Antral follicle responsiveness assessed by follicular output RaTe(FORT) correlates with follicles diameter. J Ovarian Res. 2019;12:48.

14. Iliodromiti S, Iglesias Sanchez C, Messow C-M, Cruz M, Garcia Velasco J, Nelson SM. Excessive AgeRelated Decline in Functional Ovarian Reserve in Infertile Women: Prospective Cohort of 15,500 Women. J Clin Endocrinol Metab. 2016;101:3548-54.

15. WHO | WHO laboratory manual for the examination and processing of human semen [Internet]. 5th ed. Geneva: World Health Organization; 2010 [cited 2019 Sep 9]. Available from: http://www.who.int/reproductivehealth/publications/infertility/9789241547789/en/

16. Holte J, Berglund L, Milton K, Garello C, Gennarelli G, Revelli A, et al. Construction of an evidencebased integrated morphology cleavage embryo score for implantation potential of embryos scored and transferred on day 2 after oocyte retrieval. Hum Reprod. 2007;22:548-57. 
17. Ferraretti AP, La Marca A, Fauser BCJM, Tarlatzis B, Nargund G, Gianaroli L, et al. ESHRE consensus on the definition of "poor response" to ovarian stimulation for in vitro fertilization: the Bologna criteria. Hum Reprod. 2011;26:1616-24.

18. Akaike H. A new look at the statistical model identification. IEEE Transactions on Automatic Control. 1974;19:716-23.

19. Wei LJ, Lin DY, Weissfeld L. Regression Analysis of Multivariate Incomplete Failure Time Data by Modeling Marginal Distributions. Journal of the American Statistical Association. [American Statistical Association, Taylor \& Francis, Ltd.]; 1989;84:1065-73.

20. Peduzzi P, Concato J, Kemper E, Holford TR, Feinstein AR. A simulation study of the number of events per variable in logistic regression analysis. J Clin Epidemiol. 1996;49:1373-9.

21. van Smeden M, Moons KG, de Groot JA, Collins GS, Altman DG, Eijkemans MJ, et al. Sample size for binary logistic prediction models: Beyond events per variable criteria. Stat Methods Med Res. 2019;28:2455-74.

22. Mutlu MF, Erdem M, Erdem A, Yildiz S, Mutlu I, Arisoy O, et al. Antral follicle count determines poor ovarian response better than anti-Müllerian hormone but age is the only predictor for live birth in in vitro fertilization cycles. J Assist Reprod Genet. 2013;30:657-65.

23. Tal R, Seifer DB. Ovarian reserve testing: a user's guide. Am J Obstet Gynecol. 2017;217:129-40.

24. Poseidon Group (Patient-Oriented Strategies Encompassing IndividualizeD Oocyte Number), Alviggi C, Andersen CY, Buehler K, Conforti A, De Placido G, et al. A new more detailed stratification of low responders to ovarian stimulation: from a poor ovarian response to a low prognosis concept. Fertil Steril. 2016;105:1452-3.

25. Grynberg M, Labrosse J. Understanding Follicular Output Rate (FORT) and its Implications for POSEIDON Criteria. Front Endocrinol. 2019;10:246.

26. Weghofer A, Barad DH, Darmon SK, Kushnir VA, Albertini DF, Gleicher N. The ovarian sensitivity index is predictive of live birth chances after IVF in infertile patients. Hum Reprod Open. 2020;2020:hoaa049.

27. Broer SL, van Disseldorp J, Broeze KA, Dolleman M, Opmeer BC, Bossuyt P, et al. Added value of ovarian reserve testing on patient characteristics in the prediction of ovarian response and ongoing pregnancy: an individual patient data approach. Hum Reprod Update. 2013;19:26-36.

28. Huang Y, Li J, Zhang F, Liu Y, Xu G, Guo J, et al. Factors affecting the live-birth rate in women with diminished ovarian reserve undergoing IVF-ET. Arch Gynecol Obstet. 2018;298:1017-27.

29. Peres Fagundes PA, Chapon R, Olsen PR, Schuster AK, Mattia MMC, Cunha-Filho JS. Evaluation of three-dimensional SonoAVC ultrasound for antral follicle count in infertile women: its agreement with conventional two-dimensional ultrasound and serum levels of anti-Müllerian hormone. Reprod Biol Endocrinol. 2017;15:96.

30. Broekmans FJ. Individualization of FSH Doses in Assisted Reproduction: Facts and Fiction. Front Endocrinol (Lausanne). 2019;10:181. 
31. van Tilborg TC, Oudshoorn SC, Eijkemans MJC, Mochtar MH, van Golde RJT, Hoek A, et al. Individualized FSH dosing based on ovarian reserve testing in women starting IVF/ICSI: a multicentre trial and cost-effectiveness analysis. Hum Reprod. 2017;32:2485-95.

32. Allegra A, Marino A, Volpes A, Coffaro F, Scaglione P, Gullo S, et al. A randomized controlled trial investigating the use of a predictive nomogram for the selection of the FSH starting dose in IVF/ICSI cycles. Reprod Biomed Online. 2017;34:429-38.

33. Papaleo E, Zaffagnini S, Munaretto M, Vanni VS, Rebonato G, Grisendi V, et al. Clinical application of a nomogram based on age, serum FSH and $\mathrm{AMH}$ to select the FSH starting dose in IVF/ICSI cycles: a retrospective two-centres study. Eur J Obstet Gynecol Reprod Biol. 2016;207:94-9.

34. Dessolle L, Leperlier F, Biau DJ, Fréour T, Barrière P. Proficiency in oocyte retrieval assessed by the learning curve cumulative summation test. Reprod Biomed Online. 2014;29:187-92.

\section{Figures}

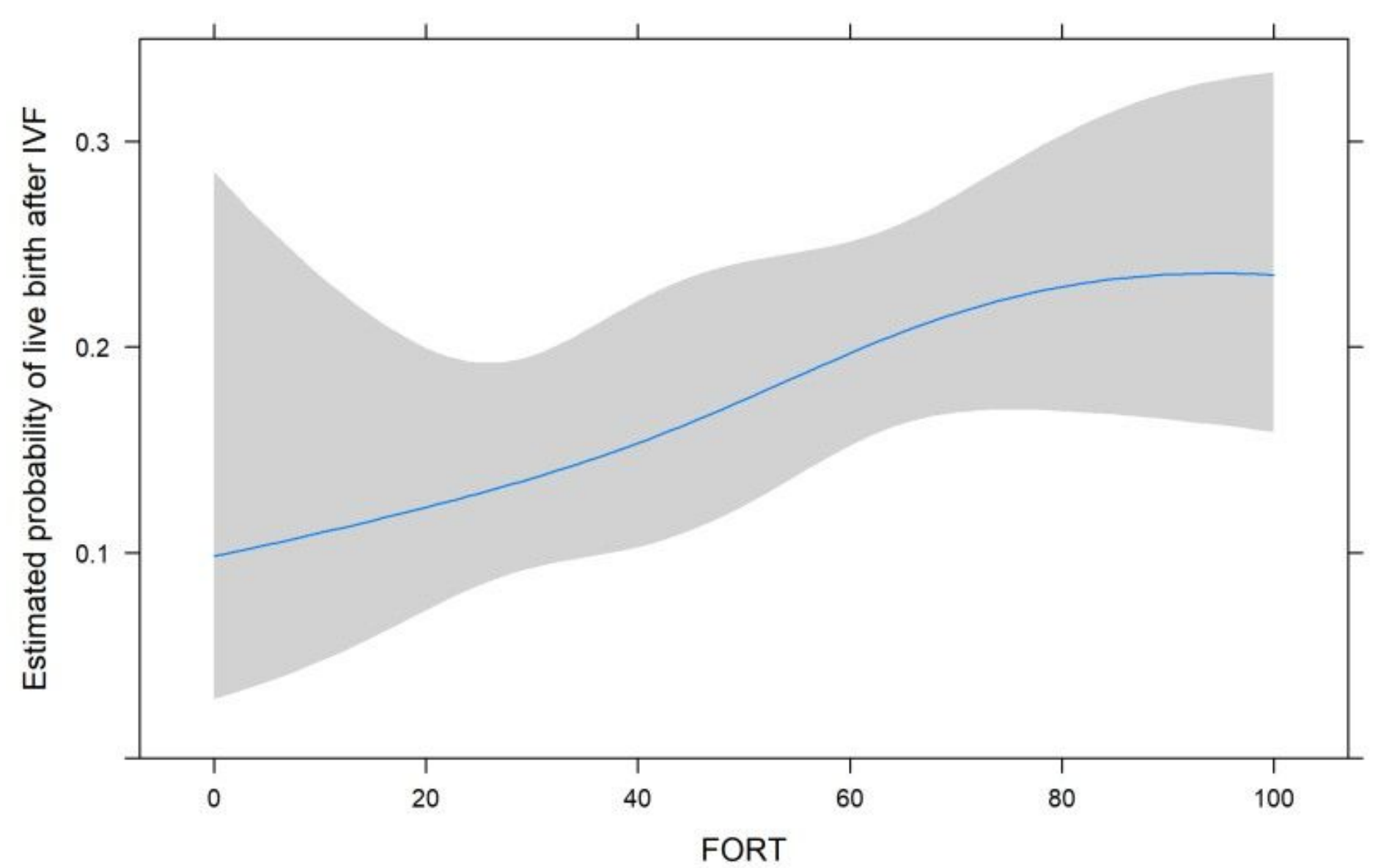

\section{Figure 1}

FORT and live birth The non-linear associations between live birth and FORT 


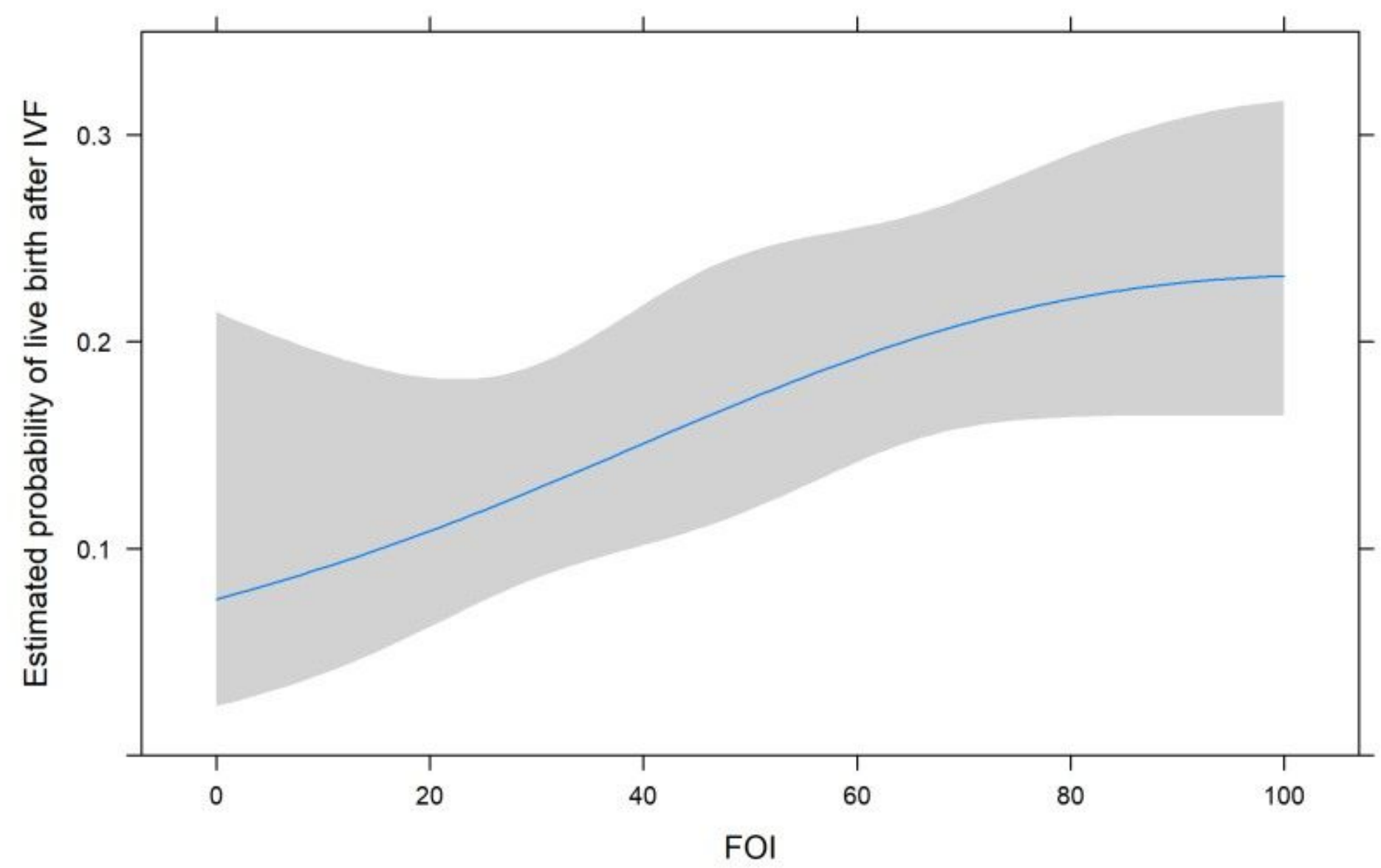

Figure 2

FOI and live birth The non-linear associations between live birth and FOI 


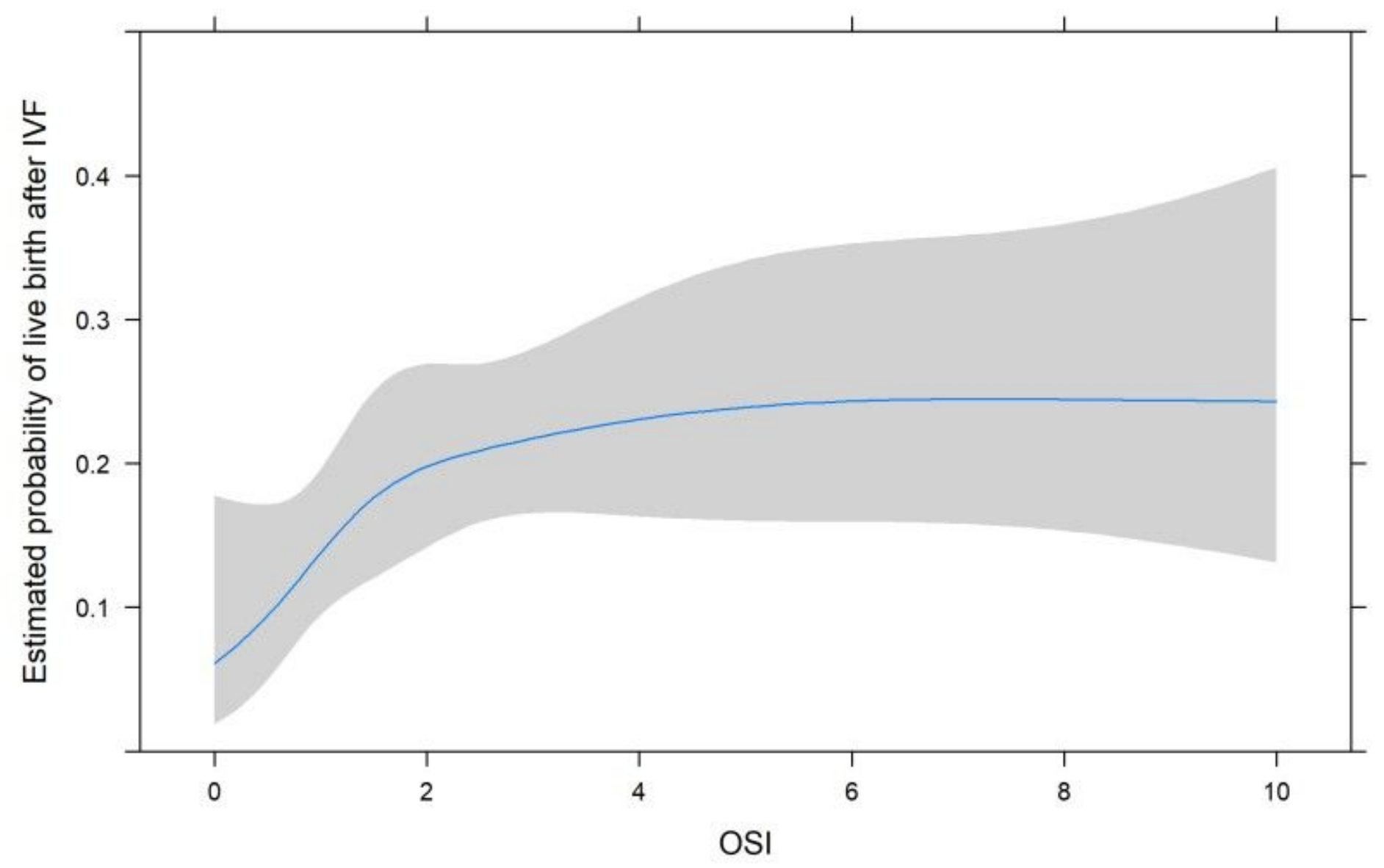

Figure 3

OSI and live birth The non-linear associations between live birth and OSI 